\title{
Unbounded Motions of Perturbed Isochronous Hamiltonian Systems at Resonance
}

\author{
Christian Fabry \\ Institut de Mathématique pure et appliquée \\ Université catholique de Louvain \\ Chemin du Cyclotron 2, Louvain-la-Neuve, B-1348 Belgium \\ e-mail: fabry@math.ucl.ac.be \\ Alessandro Fonda \\ Dipartimento di Matematica e Informatica \\ Università degli Studi di Trieste \\ P.le Europa 1, Trieste, I-34127 Italy \\ e-mail: fondass@units.it \\ Received 10 January 2005 \\ Communicated by Rafael Ortega
}

\begin{abstract}
Large amplitude solutions of asymptotically positively homogeneous perturbations of hamiltonian systems at resonance can be unbounded, either in the past, or in the future. We present conditions for boundedness or unboundedness, generalizing in particular the results obtained by Alonso and Ortega [1] for scalar second order equations with asymmetric nonlinearities.
\end{abstract}

1991 Mathematics Subject Classification. 34C11.

Key words. Unbounded solutions, isochronous hamiltonian systems, nonlinear resonance.

\section{Introduction and main results}

Asymmetric oscillators modelled by second order scalar equations of the form

$$
x^{\prime \prime}+\mu x^{+}-\nu x^{-}=g(t, x)
$$


where $\mu$ and $\nu$ are positive real numbers, $x^{+}=\max \{x, 0\}$ and $x^{-}=\max \{-x, 0\}$, despite their apparent simplicity, may surprisingly have a dynamics with a very rich structure. Assuming the function $g$ to be periodic in its first variable, with a period $T$, many authors dedicated their efforts to particular aspects of it, e.g. periodic solutions $[3,4,5,6,11,13]$, bounded solutions $[14,15]$, or unbounded solutions $[1,2,8]$.

In the still simpler case $g(t, x)=e(t)$, denoting by $\phi(t)$ a nontrivial solution of $x^{\prime \prime}+$ $\mu x^{+}-\nu x^{-}=0$, with minimal period $\tau=\frac{\pi}{\sqrt{\mu}}+\frac{\pi}{\sqrt{\nu}}$, the $\tau$-periodic function

$$
\Phi(\theta)=\int_{0}^{T} e(t) \phi(t+\theta) d t,
$$

first introduced by Dancer in [3], happens to play a crucial role "at resonance", i.e. when $T$ is a multiple of $\tau$ (see, for instance, [9] and the references therein). With this respect, Alonso and Ortega [1] proved that, if the function $\Phi$ changes sign and only has simple zeros, then all solutions of (1.1) with sufficiently large amplitude are unbounded, either in the future, or in the past. On the contrary, Liu [14] showed that, if the function $\Phi$ has a constant sign and $e(t)$ is sufficiently smooth, then all the solutions of (1.1) are bounded, both in the future and in the past.

In the present paper, following [7, 10], we are interested in studying the boundedness of the solutions of the more general system

$$
J \dot{u}=\nabla H(u)+f(t, u) .
$$

Here, $J=\left(\begin{array}{rr}0 & -1 \\ 1 & 0\end{array}\right)$ is the standard symplectic matrix, the function $H: \mathbb{R}^{2} \rightarrow \mathbb{R}$ is of class $C^{1}$, with locally Lipschitz continuous gradient, positively homogeneous of degree 2 and positive. Precisely, for every $u \in \mathbb{R}^{2}$ and $\lambda>0$,

$$
H(\lambda u)=\lambda^{2} H(u)
$$

and

$$
\min _{\|u\|=1} H(u)>0
$$

The origin is thus an isochronous center for the autonomous system

$$
J \dot{u}=\nabla H(u)
$$

all solutions of (1.5) are periodic with the same minimal period, which will be denoted by $\tau$. In this paper, we assume throughout that

$$
T \text { is an integer multiple of } \tau \text {. }
$$

In this situation, the autonomous system (1.5) has nontrivial $T$-periodic solutions, and it is said that the system (1.2) is "at resonance".

The function $f: \mathbb{R} \times \mathbb{R}^{2} \rightarrow \mathbb{R}^{2}$ is assumed to be continuous, $T$-periodic in its first variable, locally Lipschitz continuous in its second variable, and asymptotically positively homogeneous of some degree $\beta \in[0,1[$, in the following sense. 
We assume that, for some $\alpha>0$,

$$
\|f(t, u)\| \leq \alpha\left(\|u\|^{\beta}+1\right),
$$

for every $t \in \mathbb{R}$ and $u \in \mathbb{R}^{2}$. A finite number of directions being given,

$$
\alpha_{1}<\alpha_{2}<\ldots<\alpha_{l}<\alpha_{l+1}=\alpha_{1}+2 \pi,
$$

we define the set $\Sigma=\left\{\rho e^{i \alpha_{k}}: \rho \geq 0, k=1,2, \ldots, l\right\}$, which is made of $l$ rays starting from the origin. We assume that there exists a continuous function $F: \mathbb{R} \times\left(\mathbb{R}^{2} \backslash \Sigma\right) \rightarrow \mathbb{R}^{2}$ such that

$$
F(t, u)=\lim _{\lambda \rightarrow+\infty} \frac{f(t, \lambda u)}{\lambda^{\beta}},
$$

the above limit being uniform with respect to $(t, u)$ when $u$ varies in compact subsets of $\mathbb{R}^{2} \backslash \Sigma$. It is easy to see that

$$
\|F(t, u)\| \leq \alpha\|u\|^{\beta}, \quad F(t, \lambda u)=\lambda^{\beta} F(t, u),
$$

for every $t \in \mathbb{R}, u \in \mathbb{R}^{2} \backslash \Sigma$, and $\lambda>0$.

It is useful to fix a reference solution of the autonomous system (1.5). Let $\varphi: \mathbb{R} \rightarrow \mathbb{R}^{2}$ be such that

$$
J \dot{\varphi}(t)=\nabla H(\varphi(t)), \quad \text { and } \quad H(\varphi(t))=\frac{1}{2},
$$

for every $t \in \mathbb{R}$. In order to describe our main results, we introduce like in [7] the two functions

$$
\begin{aligned}
& \Phi(\theta)=\int_{0}^{T}\langle F(t, \varphi(t+\theta)) \mid \varphi(t+\theta)\rangle d t, \\
& \Psi(\theta)=\int_{0}^{T}\langle F(t, \varphi(t+\theta)) \mid \dot{\varphi}(t+\theta)\rangle d t .
\end{aligned}
$$

Because of the properties of $\varphi$, the set $\{t \in[0, \tau]: \varphi(t) \in \Sigma\}$ is finite, so that, by (1.8), $\Phi$ and $\Psi$ are well defined and they are continuous and $\tau$-periodic.

We first state a result in the case when the function $\Phi$ does not change sign.

Theorem 1.1 Assume $\Phi$ is of constant sign. If $\int_{0}^{\tau} \frac{\Psi(\theta)}{\Phi(\theta)} d \theta$ has the same sign as $\Phi$, then all solutions of (1.2) are bounded in the future, and those with sufficiently large amplitude are unbounded in the past. On the contrary, if $\Phi$ and $\int_{0}^{\tau} \frac{\Psi(\theta)}{\Phi(\theta)} d \theta$ have opposite signs, then all solutions of (1.2) are bounded in the past, and those with sufficiently large amplitude are unbounded in the future.

Our next theorem deals with the opposite situation when the function $\Phi$ does change sign. If $\omega$ is an isolated zero of $\Phi$, we denote by $\operatorname{ind}_{\Phi}(\omega)$ the index of $\Phi$ at that point, i.e. $\operatorname{ind}_{\Phi}(\omega)=+1$ if $\Phi$ goes from negative to positive values, $\operatorname{ind}_{\Phi}(\omega)=-1$ if $\Phi$ goes from positive to negative values, $\operatorname{ind}_{\Phi}(\omega)=0$ otherwise. 
Theorem 1.2 Assume that $\Phi$ changes sign, with isolated zeros

$$
\omega_{1}<\omega_{2}<\ldots<\omega_{2 m}<\omega_{2 m+1}=\omega_{1}+\tau,
$$

such that $\operatorname{ind}_{\Phi}\left(\omega_{j}\right) \neq 0$ and $\Psi\left(\omega_{j}\right) \neq 0$ for every $j$ and, letting $c_{j}=\frac{\omega_{j}+\omega_{j+1}}{2}$,

$$
\int_{\omega_{j}}^{c_{j}} \frac{d \theta}{|\Phi(\theta)|}=\int_{c_{j}}^{\omega_{j+1}} \frac{d \theta}{|\Phi(\theta)|}=+\infty .
$$

Assume moreover that one of the following two situations hold:

(a) $\Psi\left(\omega_{j}\right)<0$, for all $\omega_{j}$ such that $\operatorname{ind} d_{\Phi}\left(\omega_{j}\right)=-1$,

(b) $\Psi\left(\omega_{j}\right)>0$, for all $\omega_{j}$ such that ind $d_{\Phi}\left(\omega_{j}\right)=+1$.

Then, all solutions of (1.2) with sufficiently large amplitude are unbounded, either in the future, or in the past.

The above theorems correspond to two different types of dynamics. Denoting by $\mathcal{P}$ the Poincaré map for the period $T$ associated to equation (1.2), and by $\mathcal{P}^{n}$ its iterates, it will be shown that, for any $u_{0} \in \mathbb{R}^{2}$ with sufficiently large norm, the divergent orbits $\left\{\mathcal{P}^{n} u_{0}: n \geq 1\right\}$ behave, roughly speaking, as follows: in the case of Theorem 1.1, they go spiralling around the origin, while, in the case of Theorem 1.2, they tend to approach a half-line, either in the past or in the future.

As an immediate consequence of Theorem 1.2, we have the following.

Corollary 1.1 Assume that $\Phi$ is differentiable and changes sign, with simple zeros

$$
\omega_{1}<\omega_{2}<\ldots<\omega_{2 m}<\omega_{2 m+1}=\omega_{1}+\tau,
$$

and that, for every $j$,

$$
\Phi^{\prime}\left(\omega_{j}\right) \Psi\left(\omega_{j}\right)>0 .
$$

Then, all solutions of (1.2) with sufficiently large amplitude are unbounded, either in the future, or in the past.

It will be shown in the next section that this corollary generalizes in different directions some previous results by Alonso and Ortega [1], Dambrosio [2], and Kunze, Küpper and Liu [12]; these results are typically obtained in situations where $\Psi$ is the derivative of $\Phi$ (see also Yang [17], who considers a more general differential operator).

As a consequence of Theorem 1.1 and Massera's Theorem, system (1.2) always admits $T$-periodic solutions when $\Phi$ has constant sign. This has already been shown in [7], where the existence of $T$-periodic solutions was also proved in the setting of Corollary 1.1 , provided that $m \neq 1$.

Notice that, when $\Phi$ has constant sign, the boundedness of all solutions of (1.2) implies, by Theorem 1.1 , that

$$
\int_{0}^{\tau} \frac{\Psi(\theta)}{\Phi(\theta)} d \theta=0 .
$$

The converse is not true, even in the particular case $\Psi=\Phi^{\prime}$, as we show by an example in Section 6. However, in the special case where the function $f$ only depends on $t$ and is sufficiently smooth, the boundedness of all solutions has been proved e.g. in $[10,14,15]$. 
It is clear that the functions $\Phi$ and $\Psi$ play an important role in the study of the dynamics of the system (1.2), as shown also by the results on periodic solutions obtained by the authors in [7]. The proofs of our Theorems 1.1 and 1.2, in Section 5, will give much more information about the behaviour of the solutions. At the end of the paper, we will discuss an example where the sign is reversed with respect to the inequality (1.11), and the dynamics seems to be rather complicated.

The arguments treated in this paper can certainly be adapted in order to deal with some more general differential operators, like the so-called "p-laplacian" type operators (see e.g. [17]). However, for simplicity reasons, we prefer to deal only with this more elementary situation.

\section{Some examples}

In this section we show how to compute the functions $\Phi$ and $\Psi$ in some special situations.

When $F(t, u)$ is of the form

$$
F(t, u)=G(u)+p(t)
$$

we have

$$
\Phi(\theta)=\kappa_{1}+\int_{0}^{T}\langle p(t) \mid \varphi(t+\theta)\rangle d t, \quad \Psi(\theta)=\kappa_{2}+\int_{0}^{T}\langle p(t) \mid \dot{\varphi}(t+\theta)\rangle d t,
$$

where

$$
\kappa_{1}=\int_{0}^{T}\langle G(\varphi(t)) \mid \varphi(t)\rangle d t, \quad \kappa_{2}=\int_{0}^{T}\langle G(\varphi(t)) \mid \dot{\varphi}(t)\rangle d t .
$$

Then, $\Psi(\theta)=\Phi^{\prime}(\theta)+\kappa_{2}$. The application of Theorem 1.1 is rather easy in this case, if $\Phi$ has constant sign. Since

$$
\int_{0}^{\tau} \frac{\Psi(s)}{\Phi(s)} d s=\int_{0}^{\tau} \frac{\Phi^{\prime}(s)}{\Phi(s)} d s+\int_{0}^{\tau} \frac{\kappa_{2}}{\Phi(s)} d s=\kappa_{2} \int_{0}^{\tau} \frac{d s}{\Phi(s)}
$$

the sign of $\kappa_{2}$ will decide the unboundedness in the future or in the past of the large amplitude solutions.

\section{Corollary 2.1 Assume}

$$
\sup \left\{\int_{0}^{T}\langle p(t) \mid \varphi(t+\theta)\rangle d t: \theta \in[0, \tau]\right\}<\left|\kappa_{1}\right|
$$

If $\kappa_{2}>0$, then all solutions of (1.2) are bounded in the future, and those with sufficiently large amplitude are unbounded in the past. On the contrary, if $\kappa_{2}<0$, then all solutions of (1.2) are bounded in the past, and those with sufficiently large amplitude are unbounded in the future. 
As a particular case, consider the scalar second order equation

$$
x^{\prime \prime}+\mu x^{+}-\nu x^{-}+g\left(x, x^{\prime}\right)=e(t) .
$$

Here, we take

$$
H(x, y)=\frac{1}{2}\left[\mu\left(x^{+}\right)^{2}+\nu\left(x^{-}\right)^{2}+y^{2}\right],
$$

with $\mu>0$ and $\nu>0$. We can choose $\varphi(t)=\left(\phi(t), \phi^{\prime}(t)\right)$, where $\phi(t)$ is a nontrivial solution of $x^{\prime \prime}+\mu x^{+}-\nu x^{-}=0$. Such a solution is periodic with minimal period $\tau=$ $\frac{\pi}{\sqrt{\mu}}+\frac{\pi}{\sqrt{\nu}}$, and can be written explicitly:

$$
\phi(t)= \begin{cases}\frac{1}{\sqrt{\mu}} \sin (\sqrt{\mu} t), & \text { if } t \in\left[0, \frac{\pi}{\sqrt{\mu}}\right] \\ -\frac{1}{\sqrt{\nu}} \sin \left(\sqrt{\nu}\left(t-\frac{\pi}{\sqrt{\mu}}\right)\right), & \text { if } t \in\left[\frac{\pi}{\sqrt{\mu}}, \frac{\pi}{\sqrt{\mu}}+\frac{\pi}{\sqrt{\nu}}\right] .\end{cases}
$$

The function $g(x, y)$ is assumed to be locally Lipschitz continuous, and the function $e(t)$ continuous and $T$-periodic. Let $N$ be the integer for which $T=N \tau$. We illustrate by an example for which $\beta=0$ in (1.6) and (1.7), with $l=4$ and $\alpha_{1}=0, \alpha_{2}=\frac{\pi}{2}, \alpha_{3}=\pi$, and $\alpha_{4}=\frac{3 \pi}{2}$.

We deal with a Liénard - Rayleigh equation of the type

$$
x^{\prime \prime}+h_{1}(x) x^{\prime}+h_{2}\left(x^{\prime}\right)+\mu x^{+}-\nu x^{-}+h_{3}(x)=e(t) .
$$

Denoting by $H_{1}$ a primitive of $h_{1}$, the equation can be written as

$$
J\left(\begin{array}{l}
x^{\prime} \\
y^{\prime}
\end{array}\right)=\nabla H\left(\begin{array}{l}
x \\
y
\end{array}\right)+\left(\begin{array}{c}
h_{2}(y)+h_{3}(x) \\
H_{1}(x)
\end{array}\right)-\left(\begin{array}{c}
e(t) \\
0
\end{array}\right) .
$$

Assume

$$
\begin{array}{ll}
\exists H_{1}( \pm \infty) \in \mathbb{R}: & H_{1}( \pm \infty)=\lim _{x \rightarrow \pm \infty} H_{1}(x), \\
\exists h_{2}( \pm \infty) \in \mathbb{R}: & h_{2}( \pm \infty)=\lim _{y \rightarrow \pm \infty} h_{2}(y), \\
\exists h_{3}( \pm \infty) \in \mathbb{R}: & h_{3}( \pm \infty)=\lim _{x \rightarrow \pm \infty} h_{3}(x) .
\end{array}
$$

Then,

$$
\begin{aligned}
& \Phi(\theta)=N \frac{\nu-\mu}{\mu \nu}\left(h_{2}(+\infty)+h_{2}(-\infty)\right)+ \\
& +2 N\left[\frac{h_{3}(+\infty)}{\mu}-\frac{h_{3}(-\infty)}{\nu}\right]-\int_{0}^{T} e(t) \phi(t+\theta) d t, \\
& \Psi(\theta)=N\left(\frac{1}{\sqrt{\mu}}+\frac{1}{\sqrt{\nu}}\right)\left(h_{2}(+\infty)-h_{2}(-\infty)\right)+ \\
& +2 N\left[H_{1}(+\infty)-H_{1}(-\infty)\right]-\int_{0}^{T} e(t) \phi^{\prime}(t+\theta) d t .
\end{aligned}
$$


In this situation, whether $\Phi$ changes sign or has constant sign, Corollaries 1.1 or 2.1 can be applied, with

$$
\kappa_{2}=2 N\left(\frac{\tau}{2 \pi}\left[h_{2}(+\infty)-h_{2}(-\infty)\right]+H_{1}(+\infty)-H_{1}(-\infty)\right)
$$

If $\kappa_{2}=0$, our situation generalizes those treated in [1,2] and Theorem 1.2 in [12] (see also [17]). Notice that, in the above, it is not necessary to assume $\mu \neq \nu$.

Gradient systems deserve a particular attention. When $F(t, u)$ is the gradient of a function $V(t, u)$, with respect to $u$, by Euler's identity,

$$
\begin{aligned}
\Phi^{\prime}(\theta) & =\frac{d}{d \theta} \int_{0}^{T}\left\langle\nabla_{u} V(t, \varphi(t+\theta)) \mid \varphi(t+\theta)\right\rangle d t \\
& =\frac{d}{d \theta} \int_{0}^{T}(\beta+1) V(t, \varphi(t+\theta)) d t \\
& =(\beta+1) \int_{0}^{T}\left\langle\nabla_{u} V(t, \varphi(t+\theta)) \mid \dot{\varphi}(t+\theta)\right\rangle d t \\
& =(\beta+1) \Psi(\theta)
\end{aligned}
$$

so that $\Psi(\theta)=(\beta+1)^{-1} \Phi^{\prime}(\theta)$. In this case, Theorem 1.1 is not applicable, but Corollary 1.1 applies when $\Phi$ changes sign, only having simple zeros.

As a particular case, let $T=\tau=2 \pi$ and $F(t, u)=\cos (n t) \nabla V(u)$, where $n$ is any positive integer. Then,

$$
\begin{aligned}
\Phi(\theta)= & \cos (n \theta) \int_{0}^{2 \pi} \cos (n t)\langle\nabla V(\varphi(t)) \mid \varphi(t)\rangle d t+ \\
& +\sin (n \theta) \int_{0}^{2 \pi} \sin (n t)\langle\nabla V(\varphi(t)) \mid \varphi(t)\rangle d t
\end{aligned}
$$

and $\Psi(\theta)=(\beta+1)^{-1} \Phi^{\prime}(\theta)$. So, if $\Phi$ does not vanish identically, it has precisely $n$ simple zeros, in which case Corollary 1.1 applies.

As a further example, consider, for $\beta \in[0,1[$, the equation

$$
x^{\prime \prime}+\mu x^{+}-\nu x^{-}+e(t) \frac{|x|^{\beta} x}{|x|+1}=0 .
$$

Let $e: \mathbb{R} \rightarrow \mathbb{R}$ be continuous and $T$-periodic, $T$ being a multiple of $\tau=\frac{\pi}{\sqrt{\mu}}+\frac{\pi}{\sqrt{\nu}}$. Then, with $l=2$ and $\alpha_{1}=\frac{\pi}{2}, \alpha_{2}=\frac{3 \pi}{2}$, we have

$$
\Phi(\theta)=\int_{0}^{T} e(t)|\phi(t+\theta)|^{\beta+1} d t,
$$

and $\Psi=(\beta+1)^{-1} \Phi^{\prime}$ 


\section{The Poincaré map}

Denote by $\mathcal{P}: \mathbb{R}^{2} \rightarrow \mathbb{R}^{2}$ the Poincaré map for the period $T$ associated to equation (1.2). It is well defined since the right hand side of (1.2) is locally Lipschitz continuous and grows at most linearly.

For any $\theta_{0} \in[0, \tau]$ and $r_{0}>0$, we write

$$
\mathcal{P}\left(r_{0} \varphi\left(\theta_{0}\right)\right)=r_{1} \varphi\left(\theta_{1}\right) ;
$$

in the sequel, we will try to evaluate $\theta_{1}$ and $r_{1}$, when $r_{0}$ is large enough.

If $u(t)$ is a solution of (1.2) with starting point $u(0)=r_{0} \varphi\left(\theta_{0}\right)$, with $r_{0}>0$, we can write

$$
u(t)=r(t) \varphi(t+\theta(t)) .
$$

As long as $r(t)>0$, the functions $\theta(t)$ and $r(t)$ are of class $C^{1}$ and satisfy

$$
r^{\prime} J \varphi(t+\theta)+r \theta^{\prime} J \dot{\varphi}(t+\theta)=f(t, r \varphi(t+\theta)) .
$$

Using Euler's identity and (1.9), the scalar products by $\varphi(t+\theta)$ and by $\dot{\varphi}(t+\theta)$, respectively, lead to the system

$$
\left\{\begin{array}{l}
\theta^{\prime}=\frac{1}{r}\langle f(t, r \varphi(t+\theta)) \mid \varphi(t+\theta)\rangle, \\
r^{\prime}=-\langle f(t, r \varphi(t+\theta)) \mid \dot{\varphi}(t+\theta)\rangle .
\end{array}\right.
$$

Denote by $\left(\theta\left(t ; \theta_{0}, r_{0}\right), r\left(t ; \theta_{0}, r_{0}\right)\right)$ the solution of (3.1) with starting point

$$
\theta\left(0 ; \theta_{0}, r_{0}\right)=\theta_{0} \in[0, \tau], \quad r\left(0 ; \theta_{0}, r_{0}\right)=r_{0}>0 .
$$

Writing briefly $r(t)$ for $r\left(t ; \theta_{0}, r_{0}\right)$ and $\theta(t)$ for $\theta\left(t ; \theta_{0}, r_{0}\right)$, by (1.6) we have, for some constants $c_{1}, c_{2}$ depending only on $\varphi$,

$$
\begin{aligned}
\left|\frac{r(t)}{r_{0}}-1\right| & =\left|\frac{1}{r_{0}} \int_{0}^{t}\langle f(s, r(s) \varphi(s+\theta(s))) \mid \dot{\varphi}(s+\theta(s))\rangle d s\right| \\
& \leq \frac{1}{r_{0}} \int_{0}^{t} \alpha\left(\|r(s) \varphi(s+\theta(s))\|^{\beta}+1\right)\|\dot{\varphi}(s+\theta(s))\| d s \\
& \leq \frac{\alpha c_{1}}{r_{0}} \int_{0}^{t}\left(r_{0}^{\beta}|| \frac{r(s)}{r_{0}} \varphi(s+\theta(s)) \|^{\beta}+1\right) d s \\
& \leq \frac{\alpha c_{1}}{r_{0}} \int_{0}^{t}\left(c_{2} r_{0}^{\beta}\left|\frac{r(s)}{r_{0}}\right|^{\beta}+1\right) d s \\
& \leq \frac{\alpha c_{1}}{r_{0}} \int_{0}^{t}\left(c_{2} r_{0}^{\beta}\left(\left|\frac{r(s)}{r_{0}}\right|+1\right)+1\right) d s \\
& \leq \frac{\alpha c_{1}}{r_{0}} \int_{0}^{t}\left(c_{2} r_{0}^{\beta}\left(\left|\frac{r(s)}{r_{0}}-1\right|+2\right)+1\right) d s \\
& \leq \alpha c_{1} c_{2} r_{0}^{\beta-1} \int_{0}^{t}\left|\frac{r(s)}{r_{0}}-1\right| d s+\left[2 \alpha c_{1} c_{2} r_{0}^{\beta-1}+\alpha c_{1} r_{0}^{-1}\right] T,
\end{aligned}
$$


so that, by Gronwall inequality,

$$
\left|\frac{r\left(t ; \theta_{0}, r_{0}\right)}{r_{0}}-1\right| \leq\left[2 \alpha c_{1} c_{2} r_{0}^{\beta-1}+\alpha c_{1} r_{0}^{-1}\right] T \exp \left(\alpha c_{1} c_{2} r_{0}^{\beta-1} t\right) .
$$

Hence,

$$
\lim _{r_{0} \rightarrow+\infty} \frac{r\left(t ; \theta_{0}, r_{0}\right)}{r_{0}}=1,
$$

uniformly with respect to $t \in[0, T]$ and $\theta_{0} \in[0, \tau]$. In particular, there is a $\Gamma_{1}>0$ such that, for $r_{0} \geq \Gamma_{1}$, we have $r(t)>0$ for every $t \in[0, T]$. Analogously one shows that

$$
\lim _{r_{0} \rightarrow+\infty} \theta\left(t ; \theta_{0}, r_{0}\right)=\theta_{0},
$$

with the same type of uniformity.

Therefore, for $r_{0} \geq \Gamma_{1}$, we can write

$$
\left\{\begin{array}{l}
\theta_{1}=\theta_{0}+\int_{0}^{T} \frac{1}{r(t)}\langle f(t, r(t) \varphi(t+\theta(t))) \mid \varphi(t+\theta(t))\rangle d t \\
r_{1}=r_{0}-\int_{0}^{T}\langle f(t, r(t) \varphi(t+\theta(t))) \mid \dot{\varphi}(t+\theta(t))\rangle d t
\end{array}\right.
$$

We now need the following.

Lemma 3.1 For $r_{0} \geq \Gamma_{1}$, we have

$$
\left\{\begin{array}{l}
\theta_{1}=\theta_{0}+r_{0}^{\beta-1}\left[\Phi\left(\theta_{0}\right)+R_{1}\left(\theta_{0}, r_{0}\right)\right], \\
r_{1}=r_{0}-r_{0}^{\beta}\left[\Psi\left(\theta_{0}\right)+R_{2}\left(\theta_{0}, r_{0}\right)\right],
\end{array}\right.
$$

where $R_{1}$ and $R_{2}$ are such that

$$
\lim _{r_{0} \rightarrow+\infty} R_{1}\left(\theta_{0}, r_{0}\right)=\lim _{r_{0} \rightarrow+\infty} R_{2}\left(\theta_{0}, r_{0}\right)=0,
$$

uniformly for $\theta_{0} \in[0, \tau]$.

Proof. We have to show that

$$
\lim _{r_{0} \rightarrow+\infty} r_{0}^{1-\beta} \int_{0}^{T} \frac{1}{r(t)}\langle f(t, r(t) \varphi(t+\theta(t))) \mid \varphi(t+\theta(t))\rangle d t=\Phi\left(\theta_{0}\right),
$$

and

$$
\lim _{r_{0} \rightarrow+\infty} r_{0}^{-\beta} \int_{0}^{T}\langle f(t, r(t) \varphi(t+\theta(t))) \mid \dot{\varphi}(t+\theta(t))\rangle d t=\Psi\left(\theta_{0}\right),
$$

uniformly with respect to $\theta_{0} \in[0, \tau]$. We prove the first one, the second being similar. We can write

$$
\begin{aligned}
r_{0}^{1-\beta} \int_{0}^{T} \frac{1}{r(t)} & \langle f(t, r(t) \varphi(t+\theta(t))) \mid \varphi(t+\theta(t))\rangle d t= \\
& =\int_{0}^{T}\left(\frac{r(t)}{r_{0}}\right)^{\beta-1}\left\langle\frac{f(t, r(t) \varphi(t+\theta(t)))}{r(t)^{\beta}} \mid \varphi(t+\theta(t))\right\rangle d t
\end{aligned}
$$


Fix $\varepsilon>0$. Corresponding to each direction $\alpha_{k}$, we consider a small sector determined by $\left[\alpha_{k}-\eta, \alpha_{k}+\eta\right]$, for some $\eta>0$. Let $\Sigma_{\eta}$ be the union of these sectors, and define

$$
A_{\eta}\left(\theta_{0}\right)=\left\{t \in[0, T]: \varphi\left(t+\theta_{0}\right) \in \Sigma_{\eta}\right\} .
$$

Writing the above integral and the one defining $\Phi\left(\theta_{0}\right)$ as

$$
\int_{0}^{T} \cdots=\int_{A_{\eta}\left(\theta_{0}\right)} \cdots+\int_{[0, T] \backslash A_{\eta}\left(\theta_{0}\right)} \cdots,
$$

by (1.8) we have that, taking $\eta$ small enough,

$$
\left|\int_{A_{\eta}\left(\theta_{0}\right)}\left\langle F\left(t, \varphi\left(t+\theta_{0}\right)\right) \mid \varphi\left(t+\theta_{0}\right)\right\rangle d t\right| \leq \int_{A_{\eta}\left(\theta_{0}\right)} \alpha\left\|\varphi\left(t+\theta_{0}\right)\right\|^{\beta}\left\|\varphi\left(t+\theta_{0}\right)\right\| d t \leq \frac{\varepsilon}{4},
$$

and, for $r_{0}$ large enough, by (1.6) and (3.2),

$$
\begin{aligned}
\mid \int_{A_{\eta}\left(\theta_{0}\right)}\left(\frac{r(t)}{r_{0}}\right)^{\beta-1} & \left\langle\frac{f(t, r(t) \varphi(t+\theta(t)))}{(r(t))^{\beta}} \mid \varphi(t+\theta(t))\right\rangle d t \leq \\
& \leq \int_{A_{\eta}\left(\theta_{0}\right)} 2 \alpha\left(\|\varphi(t+\theta(t))\|^{\beta}+1\right)\|\varphi(t+\theta(t))\| d t \leq \frac{\varepsilon}{4} .
\end{aligned}
$$

On the other hand, for $t \in[0, T] \backslash A_{\eta}\left(\theta_{0}\right)$, by (1.7), (3.2) and (3.3),

$$
\lim _{r_{0} \rightarrow+\infty}\left\langle\frac{f(t, r(t) \varphi(t+\theta(t)))}{r(t)^{\beta}} \mid \varphi(t+\theta(t))\right\rangle=\left\langle F\left(t, \varphi\left(t+\theta_{0}\right)\right) \mid \varphi\left(t+\theta_{0}\right)\right\rangle,
$$

uniformly in $t \in[0, T] \backslash A_{\eta}\left(\theta_{0}\right)$ and $\theta_{0} \in[0, \tau]$, so that, for $r_{0}$ large enough,

$$
\begin{aligned}
\mid \int_{[0, T] \backslash A_{\eta}\left(\theta_{0}\right)}\left[\left(\frac{r(t)}{r_{0}}\right)^{\beta-1}\left\langle\frac{f(t, r(t) \varphi(t+\theta(t)))}{r(t)^{\beta}} \mid \varphi(t+\theta(t))\right\rangle-\right. \\
\left.\quad-\left\langle F\left(t, \varphi\left(t+\theta_{0}\right)\right) \mid \varphi\left(t+\theta_{0}\right)\right\rangle\right] d t \mid \leq \frac{\varepsilon}{2} .
\end{aligned}
$$

So, taking $\eta$ small and $r_{0}$ large enough, for every $\theta_{0} \in[0, \tau]$ we have

$$
\left|r_{0}^{1-\beta} \int_{0}^{T} \frac{1}{r(t)}\langle f(t, r(t) \varphi(t+\theta(t))) \mid \varphi(t+\theta(t))\rangle d t-\Phi\left(\theta_{0}\right)\right| \leq \varepsilon
$$

and the lemma is thus proved.

By the change of variable $w=r^{1-\beta}$, we obtain the following.

Lemma 3.2 For $w_{0} \geq \Gamma_{1}^{1-\beta}$, we have

$$
\left\{\begin{array}{l}
\theta_{1}=\theta_{0}+w_{0}^{-1}\left[\Phi\left(\theta_{0}\right)+\tilde{R}_{1}\left(\theta_{0}, w_{0}\right)\right] \\
w_{1}=w_{0}-(1-\beta) \Psi\left(\theta_{0}\right)+\tilde{R}_{2}\left(\theta_{0}, w_{0}\right),
\end{array}\right.
$$


where $\tilde{R}_{1}$ and $\tilde{R}_{2}$ are such that

$$
\lim _{w_{0} \rightarrow+\infty} \tilde{R}_{1}\left(\theta_{0}, w_{0}\right)=\lim _{w_{0} \rightarrow+\infty} \tilde{R}_{2}\left(\theta_{0}, w_{0}\right)=0,
$$

uniformly for $\theta_{0} \in[0, \tau]$.

Proof. Assume $\beta>0$. From Lemma 3.1 we have

$$
\left\{\begin{array}{l}
\theta_{1}=\theta_{0}+w_{0}^{-1}\left[\Phi\left(\theta_{0}\right)+R_{1}\left(\theta_{0}, w_{0}^{\frac{1}{1-\beta}}\right)\right], \\
w_{1}=w_{0}\left(1-w_{0}^{-1}\left[\Psi\left(\theta_{0}\right)+R_{2}\left(\theta_{0}, w_{0}^{\frac{1}{1-\beta}}\right)\right]\right)^{1-\beta},
\end{array}\right.
$$

and, writing

$$
(1-h)^{1-\beta}=1-(1-\beta) h+\sigma(h), \quad \lim _{h \rightarrow 0} \frac{\sigma(h)}{h}=0,
$$

we have

$$
\begin{array}{r}
w_{1}=w_{0}\left(1-(1-\beta) w_{0}^{-1}\left[\Psi\left(\theta_{0}\right)+R_{2}\left(\theta_{0}, w_{0}^{\frac{1}{1-\beta}}\right)\right]+\right. \\
\left.+\sigma\left(w_{0}^{-1}\left[\Psi\left(\theta_{0}\right)+R_{2}\left(\theta_{0}, w_{0}^{\frac{1}{1-\beta}}\right)\right]\right)\right) .
\end{array}
$$

Setting

$$
\begin{aligned}
& \tilde{R}_{1}\left(\theta_{0}, w_{0}\right)=R_{1}\left(\theta_{0}, w_{0}^{\frac{1}{1-\beta}}\right) \\
& \tilde{R}_{2}\left(\theta_{0}, w_{0}\right)=(\beta-1) R_{2}\left(\theta_{0}, w_{0}^{\frac{1}{1-\beta}}\right)+\sigma\left(w_{0}^{-1}\left[\Psi\left(\theta_{0}\right)+R_{2}\left(\theta_{0}, w_{0}^{\frac{1}{1-\beta}}\right)\right]\right),
\end{aligned}
$$

the proof is easily completed.

Comparing Lemmas 3.1 and 3.2, and noticing that the assumptions of our Theorems 1.1 and 1.2 are not affected if $\Psi$ is multiplied by a constant, we may assume from now on, without loss of generality, that $\beta=0$. Therefore, we are led to study the recurrence

$$
\left\{\begin{array}{l}
\theta_{n+1}=\theta_{n}+r_{n}^{-1}\left[\Phi\left(\theta_{n}\right)+R_{1}\left(\theta_{n}, r_{n}\right)\right] \\
r_{n+1}=r_{n}-\Psi\left(\theta_{n}\right)+R_{2}\left(\theta_{n}, r_{n}\right)
\end{array}\right.
$$

defined for $r_{n} \geq \Gamma_{1}>0$, where $R_{1}$ and $R_{2}$ are such that

$$
\lim _{r \rightarrow+\infty} R_{1}(\theta, r)=\lim _{r \rightarrow+\infty} R_{2}(\theta, r)=0,
$$

uniformly for $\theta \in[0, \tau]$. 


\section{Back in time}

Recall that the Poincaré map $\mathcal{P}: \mathbb{R}^{2} \rightarrow \mathbb{R}^{2}$ is a homeomorphism, and the recurrence (3.4) is a representation of its iterates, as long as $r_{n} \geq \Gamma_{1}$.

In order to study the dynamics for negative time, we make the change of variable $v(t)=$ $u(-t)$ in (1.2). Defining

$$
\tilde{H}(w)=-H(w), \quad \tilde{f}(t, w)=-f(-t, w),
$$

we have that $v(t)$ satisfies

$$
J \dot{v}=\nabla \tilde{H}(v)+\tilde{f}(t, v) .
$$

The assumptions (1.3) and (1.4) translate as

$$
\tilde{H}(\lambda w)=\lambda^{2} \tilde{H}(w),
$$

for every $w \in \mathbb{R}^{2}$ and $\lambda>0$, and

$$
\max _{\|w\|=1} \tilde{H}(w)<0 .
$$

Moreover, setting $\psi(t)=\varphi(-t)$, from (1.9) we have

$$
J \dot{\psi}(t)=\nabla \tilde{H}(\psi(t)), \quad \text { and } \quad \tilde{H}(\psi(t))=-\frac{1}{2},
$$

for every $t \in \mathbb{R}$. We correspondingly define

$$
\tilde{F}(t, w)=\lim _{\lambda \rightarrow+\infty} \frac{\tilde{f}(t, \lambda w)}{\lambda^{\beta}}=-F(-t, w),
$$

and

$$
\begin{aligned}
& \tilde{\Phi}(\theta)=\int_{0}^{T}\langle\tilde{F}(t, \psi(t+\theta)) \mid \psi(t+\theta)\rangle d t=-\Phi(-\theta) \\
& \tilde{\Psi}(\theta)=\int_{0}^{T}\langle\tilde{F}(t, \psi(t+\theta)) \mid \dot{\psi}(t+\theta)\rangle d t=\Psi(-\theta) .
\end{aligned}
$$

We can treat the new equation (4.1) exactly as we did for (1.2). The fact that the new Hamiltonian function is negative does not affect the main features of the procedure, and, assuming $\beta=0$, we are led to the following backwards recurrence:

$$
\left\{\begin{array}{l}
\theta_{n-1}=\theta_{n}+r_{n}^{-1}\left[-\Phi\left(\theta_{n}\right)+\tilde{R}_{1}\left(\theta_{n}, r_{n}\right)\right], \\
r_{n-1}=r_{n}+\Psi\left(\theta_{n}\right)+\tilde{R}_{2}\left(\theta_{n}, r_{n}\right),
\end{array}\right.
$$

where $\tilde{R}_{1}$ and $\tilde{R}_{2}$ are such that

$$
\lim _{r \rightarrow+\infty} \tilde{R}_{1}(\theta, r)=\lim _{r \rightarrow+\infty} \tilde{R}_{2}(\theta, r)=0
$$

uniformly for $\theta \in[0, \tau]$.

Notice that, with respect to the forward recurrence (3.4), both functions $\Phi$ and $\Psi$ appear now with the opposite sign. 


\section{Proofs of the main results}

In this section, we adapt the arguments of Alonso and Ortega [1] in order to prove our Theorems 1.1 and 1.2.

We perform in (3.4) the change of variable $\rho=(\delta r)^{-1}$, thus obtaining

$$
\left\{\begin{array}{l}
\theta_{n+1}=\theta_{n}+\delta \rho_{n} \Phi\left(\theta_{n}\right)+h_{1}\left(\theta_{n}, \rho_{n} ; \delta\right), \\
\rho_{n+1}=\rho_{n}+\delta \rho_{n}^{2} \Psi\left(\theta_{n}\right)+h_{2}\left(\theta_{n}, \rho_{n} ; \delta\right),
\end{array}\right.
$$

where

$$
\lim _{\delta \rightarrow 0} \frac{h_{1}(\theta, \rho ; \delta)}{\delta}=\lim _{\delta \rightarrow 0} \frac{h_{2}(\theta, \rho ; \delta)}{\delta}=0
$$

uniformly with respect to $(\theta, \rho)$ in compact subsets of $[0, \tau] \times] 0,+\infty[$.

This last recurrence can be interpreted as a numerical scheme approximating the differential equation

$$
\left\{\begin{array}{l}
\vartheta^{\prime}=\rho \Phi(\vartheta) \\
\rho^{\prime}=\rho^{2} \Psi(\vartheta)
\end{array}\right.
$$

with $\vartheta(0)=\theta_{0}$ and $\rho(0)=\rho_{0}>0$.

Assume $\Phi\left(\theta_{0}\right) \neq 0$; then, as long as $\Phi(\vartheta(t)) \neq 0$, we can write

$$
\frac{d \rho}{d \vartheta}=\frac{\Psi(\vartheta)}{\Phi(\vartheta)} \rho
$$

whence

$$
\rho(\vartheta)=\rho_{0} \exp \left(\int_{\theta_{0}}^{\vartheta} \frac{\Psi(s)}{\Phi(s)} d s\right)
$$

and

$$
\vartheta^{\prime}=\rho_{0} \exp \left(\int_{\theta_{0}}^{\vartheta} \frac{\Psi(s)}{\Phi(s)} d s\right) \Phi(\vartheta) .
$$

Since (5.3) is an equation with separated variables, the solutions of the Cauchy problems associated to (5.2) are unique (implying convergence of numerical schemes), as long as $\Phi(\vartheta(t)) \neq 0$. One has $\rho(\vartheta)>0$ for every $\vartheta$ and, if $\rho(\vartheta)$ approaches 0 , then necessarily either $\vartheta$ approaches a zero of $\Phi$ or, if $\Phi$ is of constant sign, $\vartheta$ approaches $\pm \infty$. Since

$$
\int_{\rho_{0}}^{\rho(t)} \frac{d u}{u^{2}}=\int_{0}^{t} \frac{\rho^{\prime}(s)}{\rho^{2}(s)} d s=\int_{0}^{t} \Psi(\vartheta(s)) d s,
$$

this approach can never be made in a finite time.

We now give a brief description of the dynamics for equation (5.2). The solution $(\vartheta(t), \rho(t))$ of $(5.2)$, with starting point $(\vartheta(0), \rho(0))=\left(\theta_{0}, \rho_{0}\right)$, is defined on a maximal interval of existence $] \alpha, \beta[$, with $-\infty \leq \alpha<0<\beta \leq+\infty$.

Assume for definiteness that $\Phi\left(\theta_{0}\right)>0$. The function $\vartheta(t)$ will then be increasing, and we distinguish two situations, depending on the fact that $\Phi$ has zeros or not. 
Consider first the situation of Theorem 1.1, with $\Phi$ of constant (positive) sign; it then follows from (5.2) and (5.3) that

$$
\lim _{t \rightarrow \alpha} \vartheta(t)=-\infty, \quad \lim _{t \rightarrow \beta} \vartheta(t)=+\infty .
$$

In this case, considering $(\vartheta, \rho)$ as generalized polar coordinates, we see from (5.3) that, if $\int_{0}^{\tau} \frac{\Psi(\theta)}{\Phi(\theta)} d \theta$ is positive, the trajectory of (5.2) spirals towards infinity, when $t$ approaches $\beta$, whereas if $\int_{0}^{\tau} \frac{\Psi(\theta)}{\Phi(\theta)} d \theta$ is negative, then $\beta=+\infty$ and the trajectory spirals towards the origin as $t \rightarrow+\infty$. This situation was not considered in [1], where typically $\Psi=\Phi^{\prime}$.

In the situation of Theorem 1.2, $\Phi$ changes sign, so $\left.\theta_{0} \in\right] \omega_{j}, \omega_{j+1}[$, for some $j$. By the use of (1.10),

$$
\lim _{t \rightarrow \alpha} \vartheta(t)=\omega_{j}, \quad \lim _{t \rightarrow \beta} \vartheta(t)=\omega_{j+1} .
$$

Since $\Phi\left(\theta_{0}\right)>0$, we have $\operatorname{ind}_{\Phi}\left(\omega_{j}\right)=+1$ and $\operatorname{ind}_{\Phi}\left(\omega_{j+1}\right)=-1$. If $(a)$ holds in Theorem 1.2 , then $\Psi\left(\omega_{j+1}\right)<0$ and, by (1.10), $\int_{\vartheta_{0}}^{\omega_{j+1}} \frac{\Psi(\theta)}{\Phi(\theta)} d \theta$ diverges towards $-\infty$. It follows from (5.3) that $\beta=+\infty$ and $\rho(t)$ goes to 0 as $t \rightarrow+\infty$. Moreover, since by (1.10) also $\int_{\omega_{j}}^{\vartheta_{0}} \frac{\Psi(\theta)}{\Phi(\theta)} d \theta$ diverges, the trajectory of (5.2) remains in the sector $\left.\vartheta \in\right] \omega_{j}, \omega_{j+1}[$, for all $t \in] \alpha, \beta\left[\right.$. On the other hand, if $(b)$ holds in Theorem 1.2, then $\Psi\left(\omega_{j}\right)>0$ and, by a similar argument, we have $\alpha=-\infty$ and $\rho(t)$ goes to 0 as $t \rightarrow-\infty$. As noticed in [1], in the particular case $\Psi=\Phi^{\prime}$ the solutions for which $\Phi\left(\theta_{0}\right) \neq 0$ are defined globally.

Analogous arguments hold if $\Phi\left(\theta_{0}\right)<0$. The case $\Phi\left(\theta_{0}\right)=0$ leads to solutions where $\vartheta(t)$ is constantly equal to $\theta_{0}$, and the behaviour of $\rho(t)$ is decided by the sign of $\Psi\left(\theta_{0}\right)$.

Remembering that $\rho=(\delta r)^{-1}$, and using the fact that (5.1) is an approximation scheme for (5.2), we will give below, on the basis of the above arguments, the details of the proofs of Theorems 1.1 and 1.2.

\subsection{Proof of Theorem 1.1}

We restrict our attention to the case where $\Phi$ is positive, the other case being treated analogously. Define

$$
\eta=\int_{0}^{\tau} \frac{\Psi(\theta)}{\Phi(\theta)} d \theta
$$

We first consider the case $\eta<0$ and show that all solutions of (1.2), with sufficiently large initial conditions, are unbounded in the future.

Let $\Delta \in] 0, \frac{\tau}{2}\left[\right.$ be such that, for every $\theta_{0}, \theta_{1}$,

$$
\left|\theta_{1}-\left(\theta_{0}+\tau\right)\right| \leq \Delta \Rightarrow \int_{\theta_{0}}^{\theta_{1}} \frac{\Psi(\theta)}{\Phi(\theta)} d \theta \leq \frac{\eta}{2} .
$$

Let us fix arbitrarily a $\theta^{*} \in[0, \tau]$. By (5.4), for any $\theta_{0} \in\left[\theta^{*}-\Delta, \theta^{*}+\Delta\right]$ there are $s_{-}<t_{-}<0<t_{+}<s_{+}$such that

$$
\begin{aligned}
\vartheta\left(t_{-}\right)=\theta^{*}-\tau, & \vartheta\left(t_{+}\right)=\theta^{*}+\tau, \\
\vartheta\left(s_{-}\right) \leq \theta^{*}-\tau-2 \Delta, & \vartheta\left(s_{+}\right) \geq \theta^{*}+\tau+2 \Delta,
\end{aligned}
$$

where $(\vartheta(t), \rho(t))$ denotes the solution of (5.2) with initial point $\vartheta(0)=\theta_{0}, \rho(0)=\rho_{0}$. 
Let us fix now $\rho_{0}=1$. Taking $\Delta$ sufficiently small, by compactness, $s_{-}$and $s_{+}$can be chosen independently of $\theta_{0} \in\left[\theta^{*}-\Delta, \theta^{*}+\Delta\right]$, and, by (5.3), there is a constant $\bar{C} \geq 1$ such that, for every $t \in\left[s_{-}, s_{+}\right]$, we have

$$
\bar{C}^{-1} \leq \rho(t) \leq \bar{C} .
$$

By standard results on numerical approximation schemes, it is possible to find a $\delta_{1} \in$ ] $0,\left(2 \bar{C} \Gamma_{1}\right)^{-1}[$ such that, if $\left.\delta \in] 0, \delta_{1}\right]$, then

$$
\begin{aligned}
& \left|\theta_{n}-\vartheta(n \delta)\right| \leq \frac{\Delta}{4}, \\
& \left|\rho_{n}-\rho(n \delta)\right| \leq \min \left\{\frac{1}{2 \Gamma_{1} \delta}, \frac{1}{2}\left(1-e^{\eta / 2}\right), \frac{1}{2 \bar{C}}\right\}, \\
& \left|\theta_{n+1}-\theta_{n}\right| \leq \frac{\Delta}{4},
\end{aligned}
$$

for every $n$ such that $n \delta \in\left[s_{-}, s_{+}\right]$. By compactness, such a $\delta_{1}$ can be chosen independently of $\theta_{0} \in[0, \tau]$.

Let $n_{1}>0$ be such that

$$
n_{1} \delta \leq t_{+}<\left(n_{1}+1\right) \delta .
$$

By (5.8) and (5.10),

$$
\theta_{n_{1}}-\frac{\Delta}{4} \leq \vartheta\left(n_{1} \delta\right) \leq \vartheta\left(t_{+}\right)=\theta^{*}+\tau \leq \vartheta\left(\left(n_{1}+1\right) \delta\right) \leq \theta_{n_{1}+1}+\frac{\Delta}{4} \leq \theta_{n_{1}}+\frac{\Delta}{2},
$$

so that

$$
\left|\theta_{n_{1}}-\left(\theta^{*}+\tau\right)\right| \leq \frac{\Delta}{2}, \quad\left|\vartheta\left(n_{1} \delta\right)-\left(\theta^{*}+\tau\right)\right| \leq \Delta,
$$

and, by (5.3), (5.6) and (5.9),

$$
\rho_{n_{1}} \leq \rho\left(n_{1} \delta\right)+\left|\rho_{n_{1}}-\rho\left(n_{1} \delta\right)\right| \leq e^{\eta / 2}+\frac{1}{2}\left(1-e^{\eta / 2}\right)=\frac{1+e^{\eta / 2}}{2}<1 .
$$

Moreover, by (5.7) and (5.9),

$$
\delta \rho_{n} \leq \delta \rho(n \delta)+\delta\left|\rho_{n}-\rho(n \delta)\right| \leq \delta \bar{C}+\frac{1}{2 \Gamma_{1}} \leq \frac{1}{\Gamma_{1}},
$$

and

$$
\rho_{n} \geq \rho(n \delta)-\left|\rho_{n}-\rho(n \delta)\right| \geq \frac{1}{\bar{C}}-\frac{1}{2 \bar{C}}>0,
$$

for every $n=1,2, \ldots, n_{1}$.

Assume $r_{0} \geq \delta_{1}^{-1}$. Taking $\delta=r_{0}^{-1}$, the above argument applies and the iterates defined by (3.4) satisfy

$$
r_{n_{1}}=\left(\delta \rho_{n_{1}}\right)^{-1} \geq \frac{2}{1+e^{\eta / 2}} r_{0}>r_{0},
$$

and

$$
r_{n}=\left(\delta \rho_{n}\right)^{-1} \geq \Gamma_{1},
$$


for every $n=1,2, \ldots, n_{1}$.

At this point, we repeat the previous argument once again with $\rho_{0}=1$ and $\theta_{0}=\theta_{n_{1}}-\tau$. Notice that, again, $\theta_{0} \in\left[\theta^{*}-\Delta, \theta^{*}+\Delta\right]$. Since $r_{n_{1}}>r_{0}$, taking $\delta=r_{n_{1}}^{-1}$, we still have $\left.\delta \in] 0, \delta_{1}\right]$, so that the estimates (5.8), (5.9), (5.10) still hold, for every $n$ such that $n \delta \in\left[s_{-}, s_{+}\right]$. We then find a $n_{2}>n_{1}$ for which

$$
\left|\theta_{n_{2}}-\left(\theta^{*}+2 \tau\right)\right| \leq \Delta
$$

and

$$
r_{n_{2}} \geq \frac{2}{1+e^{\eta / 2}} r_{n_{1}} \geq\left(\frac{2}{1+e^{\eta / 2}}\right)^{2} r_{0} .
$$

Iterating, we find an increasing sequence $\left(n_{k}\right)_{k}$, such that

$$
\left|\theta_{n_{k}}-\left(\theta^{*}+k \tau\right)\right| \leq \Delta
$$

and

$$
r_{n_{k}} \geq\left(\frac{2}{1+e^{\eta / 2}}\right)^{k} r_{0}
$$

so that

$$
\lim _{k \rightarrow+\infty} r_{n_{k}}=+\infty
$$

Moreover, for $n=n_{k}+1, n_{k}+2, \ldots, n_{k+1}$, using (5.7) and (5.9), we see that $\rho_{n} \leq \bar{C}+1$, so that

thus leading to

$$
r_{n} \geq \frac{1}{\bar{C}+1} r_{n_{k}},
$$

$$
\lim _{n \rightarrow+\infty} r_{n}=+\infty \text {. }
$$

We now consider the case $\eta>0$, and show that all solutions of (1.2) remain bounded in the future. Denote by $u\left(t ; u_{0}\right)$ the solution of $(1.2)$ with starting point $u\left(0 ; u_{0}\right)=u_{0}=$ $r_{0} \varphi\left(\theta_{0}\right)$. We can thus consider the iterates of the Poincare map applied at $u_{0}$, and obtain the points $u\left(n T ; u_{0}\right)=u_{n}=r_{n} \varphi\left(\theta_{n}\right)$, which are represented by the recurrence (3.4) if $r_{n} \geq \Gamma_{1}$.

Adapting the arguments above, it is possible to find a constant $\Gamma_{2} \geq \Gamma_{1}$ such that, if $r_{0}>\Gamma_{2}$, then, as long as $r_{n}$ remains greater that $\Gamma_{2}$, the inequality

$$
r_{n} \leq(\bar{C}+1) r_{0}
$$

holds, so that $\left\|u_{n}\right\| \leq \bar{K}\left\|u_{0}\right\|$, for some constant $\bar{K} \geq \bar{C}+1$. Now, let $\Gamma_{3} \geq \Gamma_{2}$ be such that, if $r_{0} \leq \Gamma_{2}$, then $\left\|u_{1}\right\|=r_{1}\left\|\varphi\left(\theta_{1}\right)\right\| \leq \Gamma_{3}$. Then, we can be sure that, for any $u_{0}$, we will have

$$
\left\|u_{n}\right\| \leq \max \left\{\left\|u_{0}\right\|, \Gamma_{3}\right\} \bar{K},
$$

for every $n \geq 0$.

Concerning the boundedness or unboundedness of the solutions in the past, we are easily reconducted to the above by reversing the time and working with (4.2), as shown in Section 4. 


\subsection{Proof of Theorem $\mathbf{1 . 2}$}

The following lemma has been proved in [1] (see Propositions 2.1 and 2.2). It shows the existence of positively or negatively invariant sectors for the recurrence generated by the Poincaré map $\mathcal{P}$.

Lemma 5.1 Let $\omega_{j}$ be a zero of $\Phi$ such that ind $d_{\Phi}\left(\omega_{j}\right) \Psi\left(\omega_{j}\right)>0$. There is a $\Theta>0$ and $a \Gamma_{2} \geq \Gamma_{1}$ such that, if $r_{0} \geq \Gamma_{2}$ and $\left|\theta_{0}-\omega_{j}\right| \leq \Theta$, the following two situations arise:

(i) if ind $_{\Phi}\left(\omega_{j}\right)=-1$, then

$$
r_{n} \geq \Gamma_{1}, \quad\left|\theta_{n}-\omega_{j}\right| \leq \Theta,
$$

for every $n \geq 0$, and

$$
\lim _{n \rightarrow+\infty} r_{n}=+\infty
$$

(ii) if $\operatorname{ind}_{\Phi}\left(\omega_{j}\right)=+1$, then

$$
r_{n} \geq \Gamma_{1}, \quad\left|\theta_{n}-\omega_{j}\right| \leq \Theta,
$$

for every $n \leq 0$, and

$$
\lim _{n \rightarrow-\infty} r_{n}=+\infty
$$

Notice that $\Theta$ and $\Gamma_{2}$ can be chosen independently of $\omega_{j}$. Moreover, since $\Psi\left(\omega_{j}\right) \neq 0$ for every $j$, we can fix $\Theta$ so that

$$
2 \Theta<\min \left\{\omega_{j+1}-\omega_{j}: j=1,2, \ldots, 2 m\right\},
$$

and, for some constant $\gamma>0$,

$$
|\Psi(\theta)| \geq \gamma, \quad \text { for every } \quad \theta \in\left[\omega_{j}-\Theta, \omega_{j}+\Theta\right] .
$$

The following two lemmas describe situations where the sequence defined by (3.4) eventually enters one of the invariant sectors introduced in Lemma 5.1.

Lemma 5.2 Let $\omega_{j}, \omega_{j+1}$ be successive zeros of $\Phi$ such that ind $d_{\Phi}\left(\omega_{j+1}\right)=-1$ and $\Psi\left(\omega_{j+1}\right)<0$. There is a $\Gamma_{3} \geq \Gamma_{2}$ such that, if $r_{0} \geq \Gamma_{3}$ and

$$
\omega_{j}+\Theta<\theta_{0}<\omega_{j+1}-\Theta,
$$

one can find a $N>0$ such that $r_{n} \geq \Gamma_{2}$ for $n=1,2, \ldots, N$, and $\left|\theta_{N}-\omega_{j+1}\right| \leq \Theta$. So, as a direct consequence of Lemma 5.1,

$$
\lim _{n \rightarrow+\infty} r_{n}=+\infty
$$

Lemma 5.3 Let $\omega_{j-1}, \omega_{j}$ be successive zeros of $\Phi$ such that ind $\phi_{\Phi}\left(\omega_{j-1}\right)=-1$ and $\Psi\left(\omega_{j-1}\right)<0$. There is a $\Gamma_{4} \geq \Gamma_{2}$ such that, if $r_{0} \geq \Gamma_{4}$ and

$$
\omega_{j-1}+\Theta<\theta_{0}<\omega_{j}-\Theta,
$$


one can find a $N>0$ such that $r_{n} \geq \Gamma_{2}$ for $n=1,2, \ldots, N$, and $\left|\theta_{N}-\omega_{j-1}\right| \leq \Theta$. So, as a direct consequence of Lemma 5.1,

$$
\lim _{n \rightarrow+\infty} r_{n}=+\infty
$$

We only need to prove Lemma 5.2, since the proof of Lemma 5.3 is completely analogous.

Proof of Lemma 5.2. Let $(\vartheta(t), \rho(t))$ be the solution of (5.2), with $\vartheta(0)=\theta_{0}$ and $\rho(0)=1$. Since $\Phi\left(\theta_{0}\right)>0$, we have (5.5), and since $\Psi\left(\omega_{j+1}\right)<0$, it follows from (5.3) and (1.10) that $\beta=+\infty$ and

$$
\lim _{t \rightarrow+\infty} \rho(t)=0
$$

Moreover, by (5.3) and (5.12),

$$
0<\rho(t) \leq \bar{C}, \quad \text { for every } t \geq 0,
$$

the constant $\bar{C} \geq 1$ depending only on $\Theta$. Then, we can find a $S>1$ such that

$$
t \geq S-1 \quad \Rightarrow \quad 0<\omega_{j+1}-\vartheta(t)<\frac{\Theta}{2} \quad \text { and } \quad 0<\rho(t)<\frac{1}{2 \Gamma_{2}} .
$$

Taking $\Gamma_{3}$ large enough, $\delta=r_{0}^{-1} \leq \Gamma_{3}^{-1}$ is small enough so that the numerical scheme satisfies

$$
\left|\theta_{n}-\vartheta(n \delta)\right| \leq \frac{\Theta}{2}, \quad\left|\rho_{n}-\rho(n \delta)\right| \leq \frac{1}{2 \Gamma_{2}},
$$

for every $n$ such that $n \delta \in[0, S]$. So, if $\Gamma_{3} \geq 2 \Gamma_{2} \bar{C}$, since $\delta \Gamma_{3}=r_{0}^{-1} \Gamma_{3} \leq 1$, we have

$$
0<\delta \rho_{n} \leq \delta\left|\rho_{n}-\rho(n \delta)\right|+\delta \rho(n \delta) \leq \frac{\delta}{2 \Gamma_{2}}+\delta \bar{C} \leq \frac{1}{2 \Gamma_{2}}+\frac{\delta \Gamma_{3}}{2 \Gamma_{2}} \leq \frac{1}{\Gamma_{2}},
$$

so that

$$
r_{n}=\left(\delta \rho_{n}\right)^{-1} \geq \Gamma_{2},
$$

for all such $n$. Denoting by $N$ the integer part of $S / \delta$, it follows from the above that

$$
\left|\theta_{N}-\omega_{j+1}\right| \leq \Theta, \quad r_{N} \geq \Gamma_{2} .
$$

We will now complete the proof of Theorem 1.2. We are going to consider only the situation $(a)$, since $(b)$ can be treated by reversing the time, as shown in Section 4 . For convenience, we can think the sequence of zeros of $\Phi$ as extended by periodicity:

$$
\omega_{2 m+j}=\omega_{j}+\tau,
$$

for every $j \in \mathbb{Z}$.

For $\theta_{0} \in[0, \tau]$, let us consider three consecutive zeros of $\Phi$,

$$
\omega_{k-1}<\omega_{k}<\omega_{k+1}
$$


with possibly $\omega_{k+1}=\omega_{k-1}+\tau$, such that $\theta_{0} \in\left[\omega_{k-1}, \omega_{k+1}\right]$, and ind $\operatorname{in}_{\Phi}\left(\omega_{k}\right)=+1$, so that

$$
\operatorname{ind}_{\Phi}\left(\omega_{k-1}\right)=\operatorname{ind}_{\Phi}\left(\omega_{k+1}\right)=-1, \quad \Psi\left(\omega_{k-1}\right)<0, \quad \Psi\left(\omega_{k+1}\right)<0 .
$$

We have different possibilities. If

$$
\left|\theta_{0}-\omega_{k-1}\right| \leq \Theta, \quad \text { or } \quad\left|\theta_{0}-\omega_{k+1}\right| \leq \Theta
$$

Lemma 5.1 directly applies, leading to

$$
\lim _{n \rightarrow+\infty} r_{n}=+\infty
$$

On the other hand, if

$$
\omega_{k-1}+\Theta<\theta_{0}<\omega_{k}-\Theta, \quad \text { or } \quad \omega_{k}+\Theta<\theta_{0}<\omega_{k+1}-\Theta
$$

Lemmas 5.2 and 5.3 apply, again leading to

$$
\lim _{n \rightarrow+\infty} r_{n}=+\infty
$$

It remains to consider the case where $\left|\theta_{0}-\omega_{k}\right| \leq \Theta$. We distinguish two possibilities, depending upon the sign of $\Psi\left(\omega_{k}\right)$.

If $\Psi\left(\omega_{k}\right)>0$, Lemma 5.1 applies, and

$$
\lim _{n \rightarrow-\infty} r_{n}=+\infty
$$

If $\Psi\left(\omega_{k}\right)<0$, either $\left|\theta_{n}-\omega_{k}\right| \leq \Theta$ for every $n \geq 0$, in which case, assuming $r_{0}$ sufficiently large and using (5.11),

$$
r_{1}=r_{0}-\Psi\left(\theta_{0}\right)+R_{2}\left(\theta_{0}, r_{0}\right) \geq r_{0}-\frac{1}{2} \Psi\left(\theta_{0}\right) \geq r_{0}+\frac{\gamma}{2}
$$

so that, by recurrence,

$$
r_{n} \geq r_{0}+n \frac{\gamma}{2}
$$

which leads to

$$
\lim _{n \rightarrow+\infty} r_{n}=+\infty
$$

Or, assuming $r_{0}$ sufficiently large, there is a $\bar{n} \geq 1$ for which $\left|\theta_{n}-\omega_{k}\right| \leq \Theta$, for $n=$ $1,2, \ldots, \bar{n}-1$, and

$$
\omega_{k-1}+\Theta<\theta_{\bar{n}}<\omega_{k}-\Theta, \quad \text { or } \quad \omega_{k}+\Theta<\theta_{\bar{n}}<\omega_{k+1}-\Theta
$$

and we are back to the situation (5.13) considered previously. 


\section{Final remarks and examples}

As shown by the proof of Theorem 1.2, if $\Psi$ happens to be negative at every zero of $\Phi$, all the solutions with sufficiently large amplitude are unbounded in the future. Arguing as in the proof of Theorem 1.1, it can be seen that, in this situation, the solutions are bounded in the past. On the contrary, if $\Psi$ is positive at every zero of $\Phi$, we are in a "dissipative" case.

The conclusion of Theorem 1.1 can be made more precise. If $\int_{0}^{\tau} \frac{\Psi(\theta)}{\Phi(\theta)} d \theta$ has the same sign as $\Phi$, it can be shown that the solutions of (1.2) are indeed uniformly ultimately bounded in the future: there is a compact set $K$ in $\mathbb{R}^{2}$ such that, for every solution $u$ of (1.2), there is a $\bar{t}$ such that $u(t) \in K$ for every $t \geq \bar{t}$. Similarly, if $\int_{0}^{\tau} \frac{\Psi(\theta)}{\Phi(\theta)} d \theta$ and $\Phi$ have opposite signs, the solutions of (1.2) are uniformly ultimately bounded in the past.

We now show by an example that, even if $\Phi$ has constant sign and $\Psi=\Phi^{\prime}$, solutions can still be unbounded. Let $T=\tau=2 \pi$ and consider the equation

$$
x^{\prime \prime}+x=\arctan x+\frac{x^{\prime}}{1+\left(x^{\prime}\right)^{2}} .
$$

Proceeding as in Section 2, a simple calculation shows that

$$
\Phi(\theta)=-2 \pi, \quad \Psi(\theta)=0,
$$

for every $\theta \in \mathbb{R}$. By phase-plane analysis, it can be shown that the trajectories spiral around the origin with growing amplitude, and are unbounded in the future. Clearly, in this example, the Poincaré map is not area-preserving, an assumption which is often made to prove the boundedness of all solutions by the use of some kind of twist theorem.

All the results of this paper can be adapted to deal with the less restrictive assumption

$$
T \text { and } \tau \text { are commensurable. }
$$

Indeed, if $T=\frac{p}{q} \tau$, defining $\tilde{T}=q T$ we have that $\tilde{T}$ is an integer multiple of $\tau$, and it is sufficient to re-define the functions $\Phi$ and $\Psi$ by taking $\tilde{T}$ instead of $T$, so to recover the previous situation. The case when $T$ and $\tau$ are not commensurable has been treated, e.g. in [16].

As a last remark, we point out a particular behaviour of the solutions of (1.2) when the sign is reversed in inequality (1.11). For the sake of definiteness, assume for instance that $\operatorname{ind}_{\Phi}\left(\omega_{j}\right)=+1$ for the even values of $j$. By the arguments of Lemmas 5.2 and 5.3, if

$$
\left|\theta_{0}-\omega_{j}\right| \geq \Theta, \quad \text { for all even } j
$$

and if $r_{0}$ is sufficiently large, the sequence $\left(r_{n}\right)_{n}$ will at some stage enter one of sectors $\left[\omega_{k}-\Theta, \omega_{k}+\Theta\right]$, for some odd $k$. Adapting the arguments of the proof of Lemma 5.1 (see [1]), it can be seen that the sequence $\left(r_{n}\right)_{n}$ will decrease until it gets below some fixed value $R$. However, this does not imply that the sequence will remain bounded in the future. Indeed, it may at some point enter a sector $\left[\omega_{k}-\Theta, \omega_{k}+\Theta\right]$ for some even $k$, and later escape from it, perhaps with a large value of $r_{n}$, or it could even remain "trapped" in it, in which case $r_{n}$ will go to infinity, since $\Psi\left(\omega_{k}\right)<0$. 
To illustrate the above discussion, consider the equation

$$
x^{\prime \prime}+x=\cos t g\left(x, x^{\prime}\right) .
$$

Here $T=\tau=2 \pi, g$ is locally Lipschitz continuous and bounded, and there is a continuous function $G: \mathbb{R}^{2} \backslash \Sigma \rightarrow \mathbb{R}$ such that

$$
G(x, y)=\lim _{\lambda \rightarrow+\infty} g(\lambda x, \lambda y),
$$

the above limit being uniform with respect to $(x, y)$ in compact subsets of $\mathbb{R}^{2} \backslash \Sigma$, with $\Sigma$ defined as in the Introduction. So, $\beta=0$, and taking $\varphi(t)=(\cos t,-\sin t)$, we have that

$$
\Phi(\theta)=A \cos \theta+B \sin \theta, \quad \Psi(\theta)=-B \cos \theta-C \sin \theta,
$$

where

$$
\begin{aligned}
& A=\int_{0}^{2 \pi} \cos ^{2} t G(\cos t,-\sin t) d t, \\
& B=\int_{0}^{2 \pi} \sin t \cos t G(\cos t,-\sin t) d t, \\
& C=\int_{0}^{2 \pi} \sin ^{2} t G(\cos t,-\sin t) d t .
\end{aligned}
$$

We will assume that $A^{2}+B^{2} \neq 0$. Taking into account the form of $\Phi$ and $\Psi$, it can be checked that the conditions (1.11) of Corollary 1.1 are satisfied if $B^{2}-A C<0$, in which case we can assert that the solutions with large amplitude are unbounded, either in the past, or in the future. On the contrary, if $B^{2}-A C>0$, we are in a situation where the sign is reversed with respect to inequality (1.11).

Observe that equation (5.3) writes here

$$
\rho(\vartheta)=\rho_{0} e^{\frac{-B(A+C)}{A^{2}+B^{2}}\left(\vartheta-\theta_{0}\right)}\left|\frac{A \cos \vartheta+B \sin \vartheta}{A \cos \theta_{0}+B \sin \theta_{0}}\right|^{-\frac{B^{2}-A C}{A^{2}+B^{2}}} .
$$

Writing as usual $\mathcal{P}^{n}\left(r_{0} \varphi\left(\theta_{0}\right)\right)=r_{n} \varphi\left(\theta_{n}\right)$, we can expect, with $r_{n}=1 /\left(\delta \rho_{n}\right)$, that

$$
r_{n} \simeq r_{0} e^{\frac{B(A+C)}{A^{2}+B^{2}}\left(\theta_{n}-\theta_{0}\right)}\left|\frac{A \cos \theta_{n}+B \sin \theta_{n}}{A \cos \theta_{0}+B \sin \theta_{0}}\right|^{\frac{B^{2}-A C}{A^{2}+B^{2}}} .
$$

Let $\gamma \in[0,2 \pi[$ be such that

$$
A \cos \theta+B \sin \theta=\sqrt{A^{2}+B^{2}} \cos (\theta-\gamma),
$$

for every $\theta \in \mathbb{R}$. When $B^{2}-A C>0$, the equation

$$
r=r_{0} e^{\frac{B(A+C)}{A^{2}+B^{2}}\left(\theta-\theta_{0}\right)}\left|\frac{\cos (\theta-\gamma)}{\cos \left(\theta_{0}-\gamma\right)}\right|^{\frac{B^{2}-A C}{A^{2}+B^{2}}}
$$


represents in polar coordinates, two closed simple curves passing through the origin, one contained in the sector $\theta \in\left[\gamma-\frac{\pi}{2}, \gamma+\frac{\pi}{2}\right]$, the other one in the sector $\theta \in\left[\gamma+\frac{\pi}{2}, \gamma+\right.$ $\left.\frac{3 \pi}{2}\right]$. This means that, with initial conditions far away from the origin, the iterates of the Poincare map will follow approximatively the one of these two curves which contains the point $\left(r_{0}, \theta_{0}\right)$. This will bring, after some time, the iterates close to the origin, in a region where the approximations leading to (5.3) are no longer valid. The iterates then escape our analysis; they might remain near the origin for some time and later move away from it, following another curve of type (6.1), possibly shifting from one sector to the other.

Numerical experiments performed on the equation

$$
x^{\prime \prime}+x=\cos t\left(\frac{|x|}{\sqrt{1+x^{2}+\left(x^{\prime}\right)^{2}}}-\alpha\right)
$$

show solutions exhibiting indeed such a behaviour. Here,

$$
A=\frac{8}{3}-\pi \alpha, \quad B=0, \quad C=\frac{4}{3}-\pi \alpha,
$$

and the condition $B^{2}-A C>0$ is thus equivalent to

$$
\alpha \in] \frac{4}{3 \pi}, \frac{8}{3 \pi}[\text {. }
$$

\section{References}

[1] J. M. Alonso and R. Ortega, Roots of unity and unbounded motions of an asymmetric oscillator, J. Differential Equations 143 (1998), 201-220.

[2] W. Dambrosio, A note on the existence of unbounded solutions to a perturbed asymmetric oscillator, Nonlin. Anal. 50 (2002), 333-346.

[3] E. N. Dancer, Boundary-value problems for weakly nonlinear ordinary differential equations, Bull. Austral. Math. Soc. 15 (1976), 321-328.

[4] P. Drabek and S. Invernizzi, On the periodic boundary value problem for forced Duffing equations with jumping nonlinearity, Nonlin. Anal. 19 (1986), 643-650.

[5] C. Fabry, Landesman-Lazer conditions for periodic boundary value problems with asymmetric nonlinearities, J. Differential Equations 116 (1995), 405-418.

[6] C. Fabry and A. Fonda, Nonlinear resonance in asymmetric oscillators, J. Differential Equations 147 (1998), 58-78.

[7] C. Fabry and A. Fonda, Periodic solutions of perturbed isochronous Hamiltonian systems at resonance, J. Differential Equations 214 (2005), 299-325.

[8] C. Fabry and J. Mawhin, Oscillations of a forced asymmetric oscillator at resonance, Nonlinearity 13 (2000), 493-505.

[9] C. Fabry and J. Mawhin, Properties of solutions of some forced nonlinear oscillators at resonance. Progress in nonlinear analysis (Tianjin, 1999), 103-118, Nankai Ser. Pure Appl. Math. Theoret. Phys., 6, World Sci. Publishing, River Edge, NJ, 2000. 
[10] A. Fonda, Positively homogeneous hamiltonian systems in the plane, J. Differential Equations 200 (2004), 162-184.

[11] S. Fučík, Solvability of Nonlinear Equations and Boundary Value Problems, Reidel, Boston, 1980.

[12] M. Kunze, T. Küpper and B. Liu, Boundedness and unboundedness of solutions for reversible oscillators at resonance, Nonlinearity 14 (2001), 1105-1122.

[13] A. C. Lazer and P. J. McKenna, Existence, uniqueness and stability of oscillations in differential equations with asymmetric nonlinearities, Trans. Amer. Math. Soc. 315 (1989), 721-739.

[14] B. Liu, Boundedness in asymmetric oscillators, J. Math. Anal. Appl. 231 (1999), 355373.

[15] R. Ortega, Asymmetric oscillators and twist mappings, J. London Math. Soc. 53 (1996), 325-342.

[16] Z. Wang, Irrational rotation numbers and unboundedness of solutions of the second order differential equations with asymmetric nonlinearities, Proc. Amer. Math. Soc. 131 (2003) 523-531.

[17] X. Yang, Unboundedness of the large solutions of some asymmetric oscillators at resonance, Math. Nachr. 276 (2004), 89-102. 